\title{
Editorial Manager System for online manuscript submission to Archives of Toxicology
}

\author{
Jan G. Hengstler · Hermann M. Bolt • \\ Rosemarie Marchan
}

Published online: 4 December 2008

(C) Springer-Verlag 2008

Effective 10 December 2008, Archives of Toxicology will introduce a new electronic manuscript submission and tracking system (http://www.editorialmanager.com/atox). All authors are requested to submit their manuscripts using this Editorial Manager System. This system will be available to all individuals involved in the editorial process (editors, referees and secretarial managers).

The Editorial Manager System has several advantages over the current method of submission in that it is faster, more efficient and provides step-by-step instructions, making the submission and review processes more straightforward. Additional benefits of this system include:

- Worldwide accessibility: once an internet connection is available, submissions and revisions can be made.
- Peer-review process and availability for publication are faster: because all individuals involved have access to the same online system, information is more readily available to all.

- Secure data: all data are stored on a central server which is routinely backed-up, thus minimizing loss of data.

For additional information and to submit your article online, please visit the Springer homepage at: http://www. springeronline.com and follow the "Online Submission" link. We thank you for your submissions in the past and look forward to your future articles using our new Editorial Manager System.
J. G. Hengstler $(\square) \cdot$ H. M. Bolt $\cdot$ R. Marchan Leibniz-Institut für Arbeitsforschung an der TU Dortmund, Leibniz Research Centre for Working Environment and Human Factors (IfADo), Ardeystrasse 67, 44139 Dortmund, Germany e-mail: hengstler@ifado.de 